\title{
ENHANCEMENT OF DRIVE-MODE BANDWIDTH IN MEMS VIBRATORY GYROSCOPES UTILIZING MULTIPLE OSCILLATORS
}

\author{
Cenk Acar and Andrei Shkel \\ Mechanical and Aerospace Engineering Department, University of California Irvine \\ Irvine, CA, 92697. USA.
}

\begin{abstract}
The limitations of photolithography-based micromachining technologies defines the upper-bound on the performance and robustness of micromachined gyroscopes. Conventional gyroscope designs based on matching the drive and sense modes are extremely sensitive to variations in system parameters. Non-conventional design concepts have been reported that increase bandwidth to improve robustness, but with the expense of response gain reduction. This paper presents a new approach that may yield vibratory MEMS gyroscopes with wider drive-mode bandwidth, without significant sacrifice in response gain. The approach is based on utilizing multiple drive-mode oscillators with incrementally spaced resonant frequencies to achieve wide-bandwidth response in the drive-mode. Enhanced mode-decoupling is also achieved by distributing the linear drive-mode oscillators radially and symmetrically, to form a multi-directional linear drive-mode and a torsional sense-mode; minimizing quadrature error and zero-rate-output. The approach has been implemented on bulk-micromachined prototypes fabricated in a SOI-based process, and experimentally demonstrated.
\end{abstract}

\section{INTRODUCTION}

The tolerancing capabilities of the current photolithography processes and micro-fabrication techniques are inadequate compared to the requirements for production of high-performance inertial sensors. The resulting inherent imperfections in the mechanical structure significantly limit the performance, stability, and robustness of MEMS gyroscopes [1], [2]. Thus, fabrication and commercialization of high-performance and reliable vibratory MEMS gyroscopes have proven to be one of the most challenging tasks in the micro-domain.

The operation principle of the vast majority of existing micromachined vibratory gyroscopes relies on detection of the sinusoidal Coriolis force induced on a vibrating proof-mass. The proof-mass is driven into a constant-amplitude oscillation in the drive direction, generally at resonance. When the gyroscope is subjected to an angular rotation, the sinusoidal Coriolis force is induced in the sense direction. To achieve the maximum possible response gain, the conventional gyroscopes are generally desired to operate at or near the resonance peak of both drive and sense modes. This is typically achieved by designing and electrostatically tuning the drive and sense resonant frequencies to match [3].

The drive and sense mode matching (or near-matching) requirement in vibratory gyroscopes renders the system response very sensitive to variations in system parameters, e.g. due to fabrication imperfections and fluctuations in operating conditions, which shift the drive or sense resonant frequencies [2]. Vacuum packaging of the devices to enhance sensitivity results in extremely narrow response bandwidth, leading to much tighter mode-matching requirements. Extensive research has focused on design of symmetric drive and sense-mode suspensions for mode-matching and minimizing temperature dependence [11]. However, especially for lightly-damped devices, it is recognized by many authors that the mode-matching requirement is well beyond fabrication tolerances; and none of the symmetric designs can provide the required degree of modematching without a feedback control [4], [5].

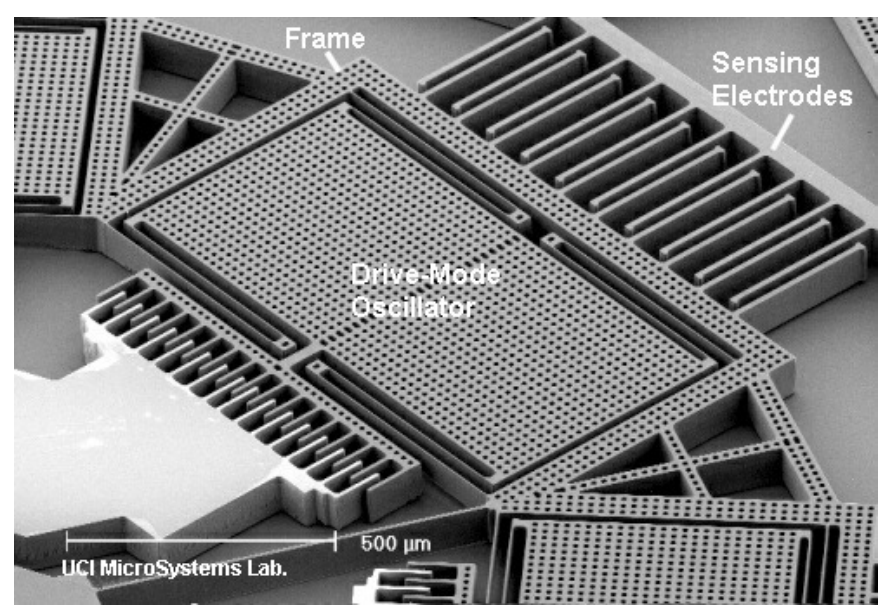

Fig. 1. SEM micrograph of a distributed-mass micromachined gyroscope prototype, utilizing multiple drive-mode oscillators with incrementally spaced resonance frequencies.

Furthermore, as the modes are matched closer, the mechanical interference between the modes becomes more significant, resulting in operation instability and drift. In order to suppress coupled oscillation and drift and to minimize the resulting zero-rate drift, various devices have been reported employing decoupled suspension systems for the drive and sense modes [9], [10]. The approach of decoupling drive and sense modes led to the first integrated commercial MEMS gyroscopes produced by Analog Devices.

The mode-matching problem and the quadrature error due to the structural imperfections are the two major challenges in MEMS gyroscope design. We have previously reported gyroscope systems that offer improved robustness by expanding the degree-of-freedom of the dynamical system [6], [7]. Even though increased-DOF gyroscope systems provide significantly increased bandwidth (over $1 \mathrm{kHz}$ ), the objective is achieved with the expense of sacrificing the response gain. This paper presents a novel approach that provides wider drive-mode bandwidth in vibratory MEMS gyroscopes with minimal sacrifice in response gain; based on utilizing multiple drivemode oscillators with incrementally spaced resonance frequencies. The concept was introduced in [8] by these authors, with the preliminary experimental results on the first generation prototypes. In this paper we generalize the approach, theoretically and experimentally explore the involved design trade-offs to achieve a wide drive-mode bandwidth, and present the experimental characterization results that demonstrate the feasibility of the design concept.

\section{THE APPROACH}

The proposed approach explores the possibility of increasing the drive-mode response bandwidth of micromachined gyroscopes, without sacrificing gain, by utilizing multiple resonators in the drivemode. Also, by distributing the drive-mode oscillators symmetrically around the center of a supporting frame, multi-directional linear drive-mode and a single torsional sense-mode are formed (Figure 2), 
effectively decoupling the drive and sense modes. Thus, zero-rateoutput and quadrature error due to mechanical mode coupling are suppressed effectively in the presence of structural imperfections. Furthermore, the sensing electrodes attached to the supporting frame do not deflect due to the drive-mode oscillations.

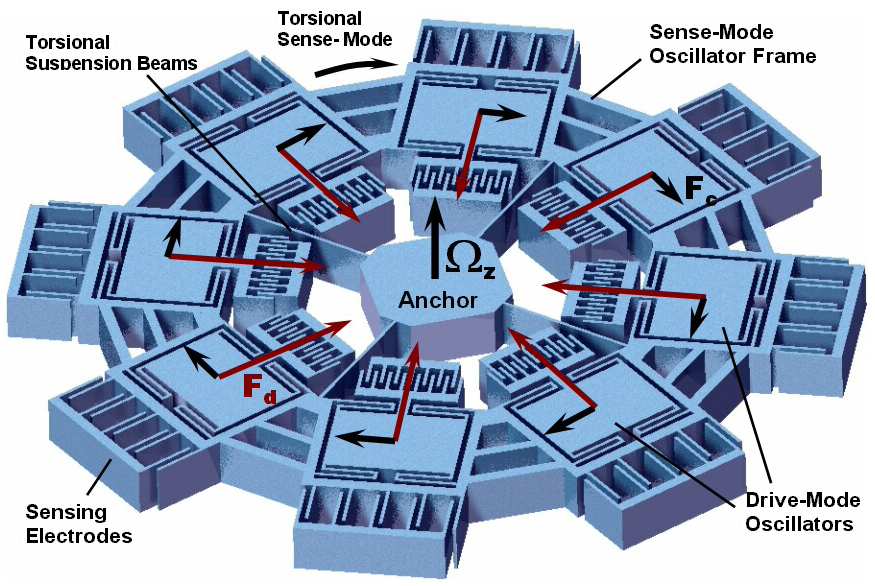

Fig. 2. Conceptual illustration of the distributed-mass gyroscope with 8 symmetric drive-mode oscillators, where $F_{d}$ and $F_{c}$ denote the drive and the Coriolis forces on each mass, respectively.

The distributed drive-mode oscillators are driven in-phase along the axes normal to the tangents of the supporting frame, and constrained in the tangential direction with respect to the supporting frame. In the presence of an angular rotation rate about the zaxis, the Coriolis force $F_{c i}=2 m_{i} \Omega_{z} \dot{x}_{i}$ is induced on each proof mass orthogonal to each drive-mode oscillation direction (Figure 2). Thus, each of the induced Coriolis force vectors lie in the tangential direction, combining to generate a resultant sinusoidal moment on the supporting frame. The net moment excites the supporting frame into torsional oscillations about the z-axis, which is detected by the sense capacitors, providing measurement of angular rate. Thus, the simplified equation of motion of the supporting frame in the sense-direction becomes

$$
I_{z} \ddot{\phi}+D_{z} \dot{\phi}+K_{z} \phi=\sum_{i=1}^{n} \vec{r}_{c i} \times \vec{F}_{c i}=\sum_{i=1}^{n} 2 r_{c} m_{i} \Omega_{z} \dot{x_{i}} \cdot \widehat{k}
$$

where $r_{c i}$ is magnitude of the position vector $\vec{r}_{c i}$ of the $i^{t h}$ oscillator center-of-mass, $\phi$ is the torsional deflection of the supporting frame, $I_{z}$ denotes the moment of inertia of the supporting frame combined with the proof masses, $D_{z}$ is the sense-mode torsional damping ratio, and $K_{z}$ is the torsional stiffness of the suspension.

\section{Wide-Bandwidth Operation}

In the presented design concept, a wide-bandwidth operation region is achieved in the drive-mode frequency response, by designing or actively tuning the resonance frequency of each drivemode oscillator to be incrementally spaced (Figure 3a). When the tangential Coriolis forces induced on each proof mass are combined, a constant-amplitude sinusoidal Coriolis moment is achieved over a wide frequency range (Figure $3 b$ ). The device is nominally operated in the flat region of the Coriolis moment frequency response. Thus, fluctuations in system parameters that shift oscillator resonance frequencies will not result in a significant change in the total Coriolis torque through the operation frequency band. If the sense-mode resonance frequency is designed to be accommodated in the same frequency band (Figure 3b), the requirement on the degree of modematching is relaxed, and robustness against structural and thermal parameter fluctuations is achieved.

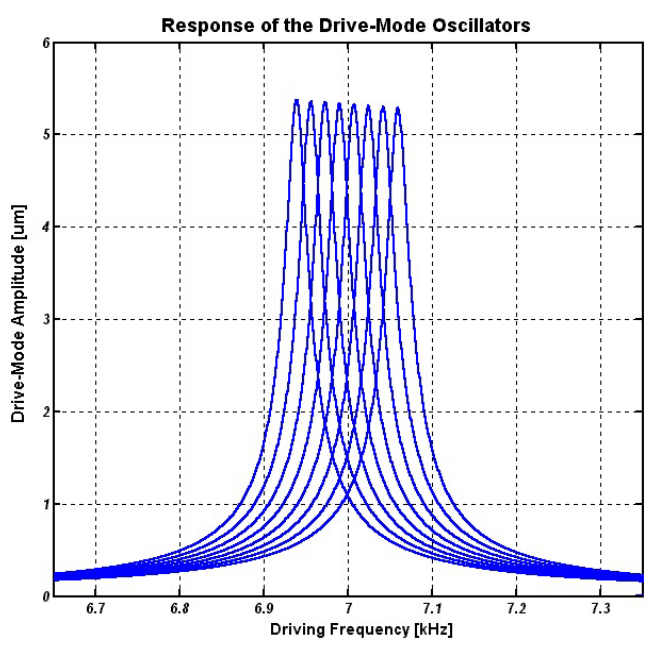

(a)

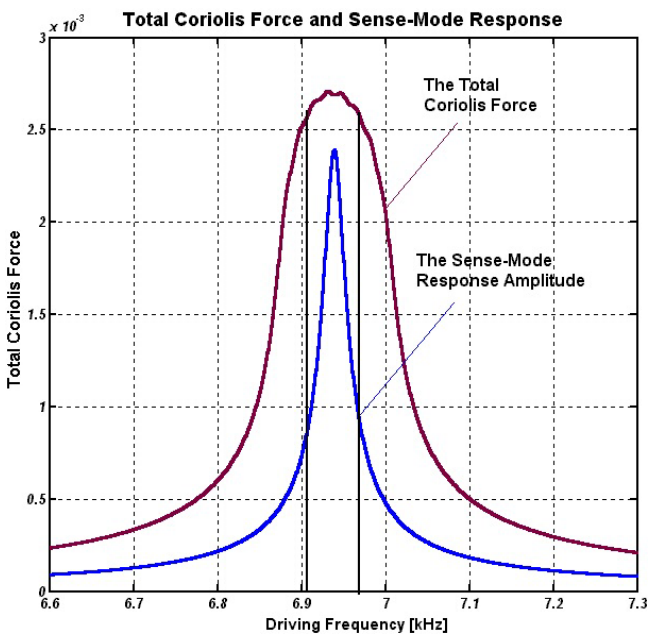

(b)

Fig. 3. (a) The frequency responses of the distributed drive-mode oscillators; (b) The frequency spectrum of the total Coriolis torque generated by the distributed drive-mode oscillators.

It should be noticed that the resonance frequency separation of the oscillators are dictated by the bandwidth of the response, which is determined by damping. In order to obtain a flat operation region in the drive-mode, the frequency separation should be less than the bandwidth of a single oscillator. If the separation of frequencies is large for high-Q resonators, the individual resonance peaks become more emphasized in the response (Figure 4), and the flat operation region will not be achieved in the drive-mode response; which is experimentally illustrated in the next section.

\section{EXPERIMENTAL ANALYSIS OF TRADE-OFFS}

\section{Fabrication of Prototypes}

The wide-bandwidth design concept was analyzed experimentally using bulk-micromachined prototype structures, fabricated in the UCI Integrated Nano-Systems Research Facility (Figure 1). Two different prototype gyroscope structures utilizing the wide-bandwidth design concept were designed: one type of structure employing comb-drive actuation to achieve large drive amplitudes, and another type employing parallel-plate actuation for a wide electrostatic tuning range.

For the fabrication of the prototypes, a one-mask process based on SOI (Silicon-on-Insulator) wafers was developed. The process relies 


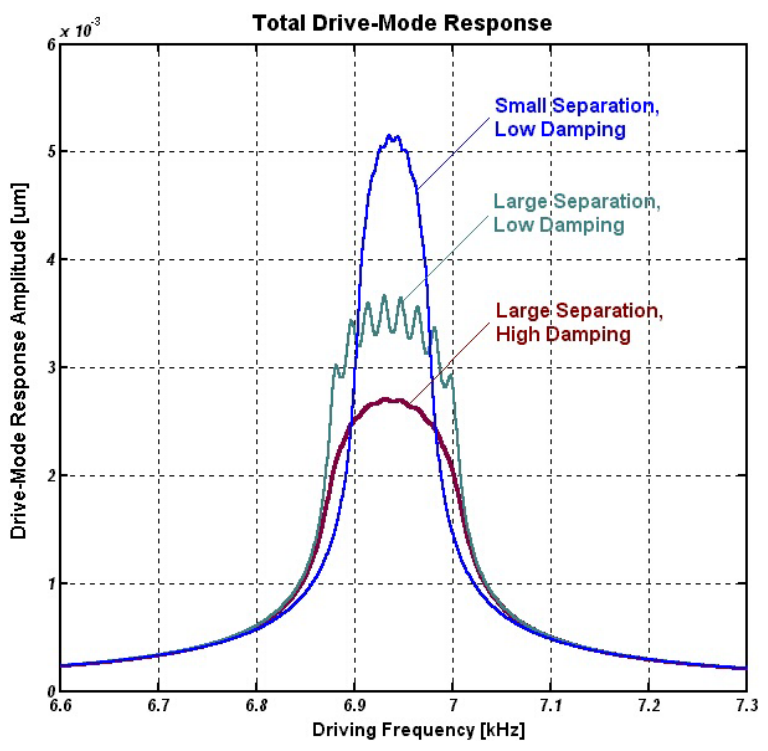

Fig. 4. The effect of damping and resonance frequency separation on the drive-mode response gain and bandwidth (effecting sensitivity and robustness, respectively).

on deep-reactive ion etching (DRIE) through the $100 \mu m$ device layer of the SOI wafer, and front-side release of the structures by etching the Oxide layer in HF solution. The process and the device design was optimized to minimize notching at the Oxide interface and excessive undercutting. In the device, $15 \mu \mathrm{m} \times 15 \mu \mathrm{m}$ holes were used to perforate the suspended structures, and $10 \mu \mathrm{m}$ gaps were used in the sensing and actuation electrodes. Anchors were designed as unperforated areas larger than $40 \mu \mathrm{m} \times 40 \mu \mathrm{m}$.

Through Finite Element Analysis simulations using the package MSC Nastran/Patran, the resonance frequency of the drive-mode oscillators were obtained at $6.98 \mathrm{kHz}$ with an elastic modulus of 130GPa for single-crystal Silicon in (100)-direction. The torsional sense mode resonance frequency of the structure about the sense axis was then located at $\omega_{z}=6.79 \mathrm{kHz}$ with four torsional suspension beams, by iteratively optimizing the beam length.

\section{Experimental Results}

The dynamic response of the linear drive-mode oscillators and the torsional sense-mode of the prototype gyroscope were characterized in a cryogenic MMR vacuum probe station. The frequency response of the prototype devices were acquired under varying pressure values and at room temperature, using off-chip sensing electronics. One-port actuation and detection was employed for characterization of the drive-mode oscillators, where a single electrode was used for both driving and sensing at the same time.

The resonance frequencies of the drive-mode resonators were observed to be scattered between $4.546 \mathrm{kHz}$ and $5.355 \mathrm{kHz}$ within a $809 \mathrm{~Hz}$ frequency band (Figure 5a). The random separation of the identically-designed drive-mode resonance frequencies resulted from the fabrication imperfections. The deviation of approximately $26 \%$ from the FEA results were attributed to lateral over-etching during DRIE, the resolution of the mask used in fabrication, and the exposure and development steps of the photolithography process.

Figure $5 \mathrm{~b}$ presents the experimentally acquired frequency responses of the Port-1 oscillator, from atmospheric pressure to 1 Torr. Least-square curves were fit to each experimental data curve for estimation of the $\mathrm{Q}$ factor and the bandwidth, which are summarized in Table 1.

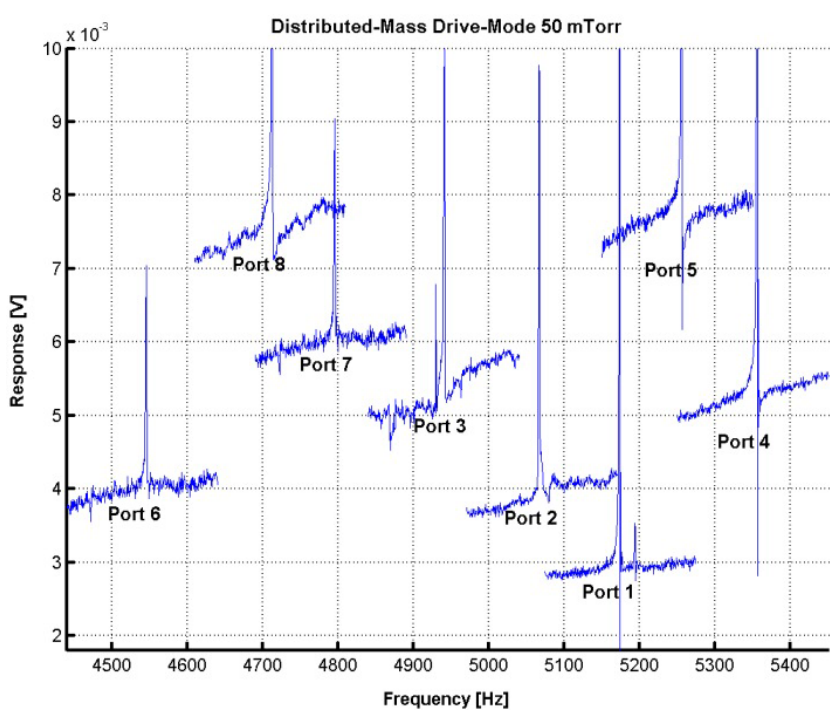

(a)

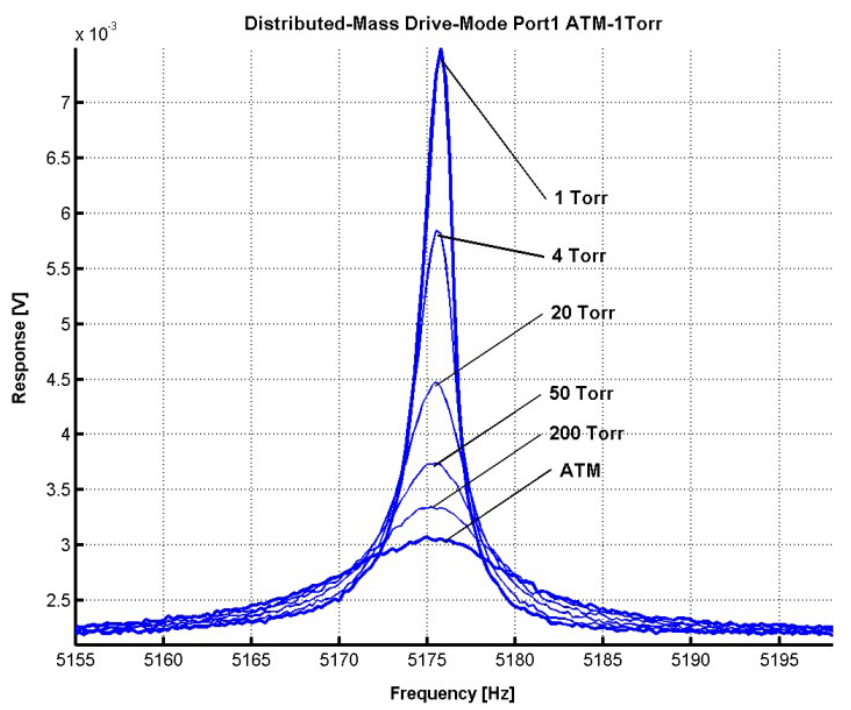

(b)

Fig. 5. (a) Experimental frequency response measurements of the 8 driveports at 50mTorr pressure; (b) Experimental measurements of the drive-mode frequency response of one of the oscillators, at different pressure values.

Drive-Mode

\begin{tabular}{|l|l|l|}
\hline Pressure & Q Factor & BW \\
\hline 1 Torr & 4187 & $1.24 \mathrm{~Hz}$ \\
\hline 4 Torr & 2912 & $1.78 \mathrm{~Hz}$ \\
\hline 20 Torr & 1861 & $2.78 \mathrm{~Hz}$ \\
\hline 50 Torr & 1339 & $3.87 \mathrm{~Hz}$ \\
\hline 200 Torr & 985 & $5.25 \mathrm{~Hz}$ \\
\hline ATM & 598 & $8.65 \mathrm{~Hz}$ \\
\hline
\end{tabular}

Table 1. The calculated $Q$ factor and the bandwidth values from the least-square curves for each pressure.

The bandwidth of the drive-mode response even at atmospheric pressure was observed to be too narrow to achieve wide-band operation without electrostatic tuning of the drive-mode frequencies. In order to achieve a wider range of electrostatic tuning, the prototype with the parallel-plate actuated drive-mode oscillators (Figure 1) was tested, and the resonance frequencies of each drivemode oscillator were electrostatically tuned to achieve uniform and smaller separation of frequencies. 
After electrostatic tuning of the parallel-plate oscillators for $10 \mathrm{~Hz}$ spacing, the close spacing of the drive-mode resonance frequencies allowed all of the resonators to be excited together, to jointly generate a resultant Coriolis moment. The total Coriolis moment, which is obtained by summing the experimentally measured response of the eight drive-ports, was observed to provide a flat range of over $140 \mathrm{~Hz}$ (Figure 6). When the experiments were repeated at reduced pressures, the resonance peaks in the flat region of the overall response became more emphasized, as was theoretically illustrated in the previous section. Based on the experimental results, it was concluded that 200 to 300 Torr is the optimal pressure for the parallel-plate devices to achieve a flat wide-bandwidth drive-mode response with $10 \mathrm{~Hz}$ spacing.

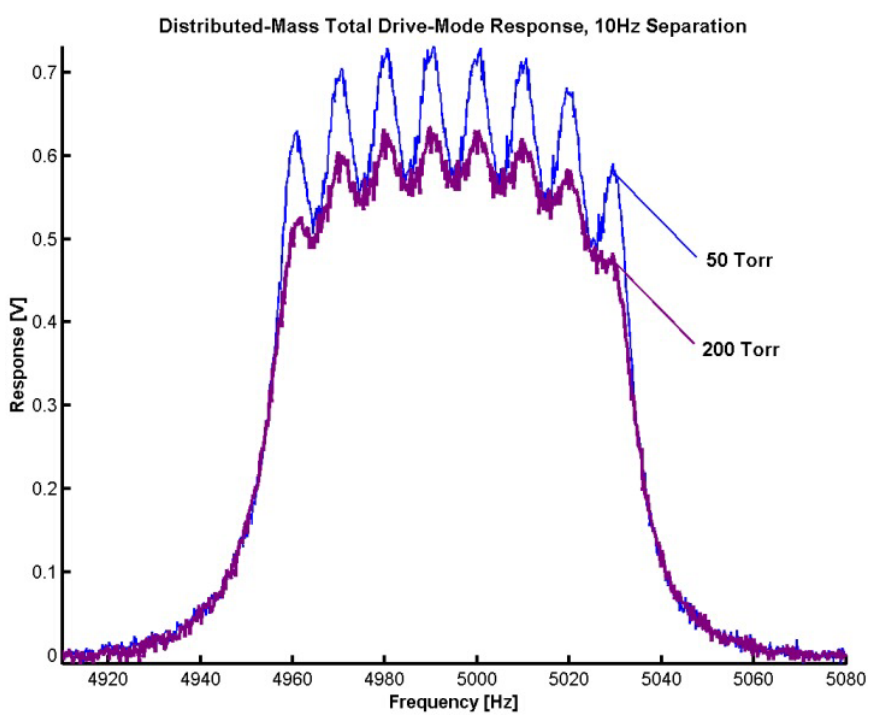

Fig. 6. Experimental frequency response measurements of the total Coriolis force induced in the sense-mode, obtained by summing the measured drivemode response of the drive ports.

The sense-mode resonance frequency of the frame was measured at $3.758 \mathrm{kHz}$ with $20 \mathrm{~V} \mathrm{DC}$ bias voltage. Figure 7 presents the experimentally acquired frequency responses of the torsional sensemode, from atmospheric pressure to 10 Torr. At pressures around 200 Torr, the parallel-plate devices were observed to provide a flat wide-bandwidth drive-mode response with individual tuning for $10 \mathrm{~Hz}$ spacing, and sufficient off-resonance sense-mode gain.

\section{CONCLUSION}

A novel design approach based on utilizing multiple drive-mode oscillators with incrementally spaced resonance frequencies was presented, which provides wider drive-mode bandwidth in vibratory MEMS gyroscopes without significant sacrifice in response gain. The operational principles were theoretically illustrated, and experimentally verified. The linear drive-mode oscillators and the torsional sense-mode of the prototype gyroscope structures were characterized under varying pressure values. The resonance frequencies of the identically-designed drive-mode resonators were observed to be scattered within a $809 \mathrm{~Hz}$ frequency band, due to the mask resolution and the fabrication imperfections. This phenomenon could be exploited in future designs, to naturally provide the required frequency separation. The bandwidth of the drive-mode response even at atmospheric pressure was observed to be too narrow to achieve wide-band operation without electrostatic tuning of the drive-mode frequencies. After electrostatic tuning of the parallel-plate oscillators for $10 \mathrm{~Hz}$ spacing, the close spacing of the drive-mode resonance

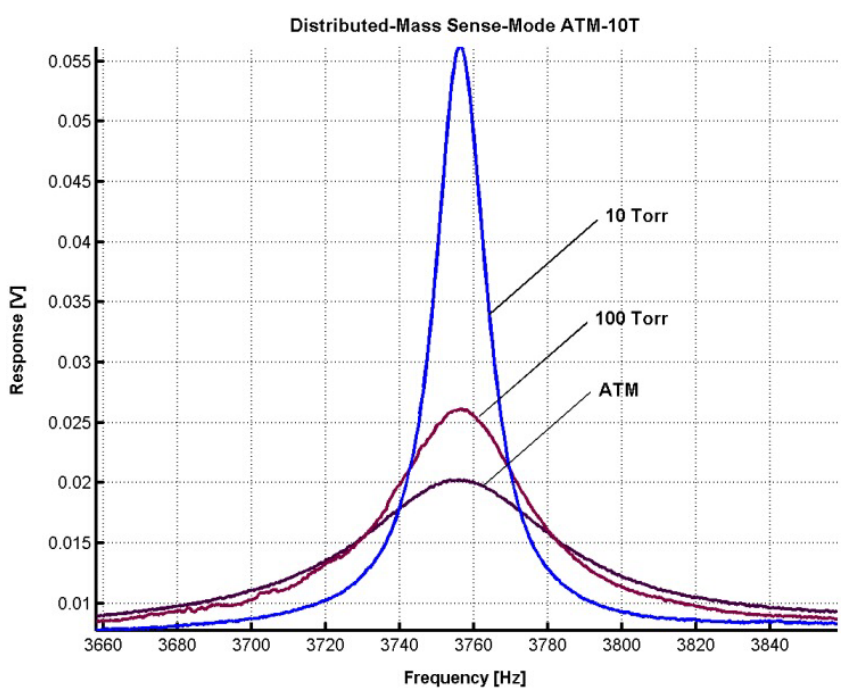

Fig. 7. Experimental measurements of the torsional sense-mode frequency response, under different pressure conditions.

frequencies allowed all of the resonators to be excited together, to jointly generate a resultant Coriolis torque. The total Coriolis torque was observed to provide a flat range of over $140 \mathrm{~Hz}$. At pressures around 200 Torr, the flat wide-bandwidth drive-mode response was achieved together with sufficient off-resonance sensemode gain, experimentally demonstrating the feasibility of the widebandwidth drive mode principle. The next generation prototypes will be fabricated using higher resolution masks to minimize random scattering of the drive-mode resonance frequencies, thus allowing to use comb-drive oscillators for larger drive-mode amplitudes.

\section{ACKNOWLEDGEMENTS}

Travel support has been generously provided by the Transducers Research Foundation and by the DARPA MEMS and DARPA BioFlips programs.

\section{REFERENCES}

[1] A. Shkel, R. Horowitz, A. Seshia, S. Park and R.T. Howe. Dynamics and Control of Micromachined Gyroscopes. American Control Conf., CA, 1999.

[2] A. Shkel, R.T. Howe, and R. Horowitz. Modeling and Simulation of Micromachined Gyroscopes in the Presence of Imperfections. Int. Conf. on Modeling and Simulation of Microsystems, 1999.

[3] W.A. Clark, R.T. Howe, and R. Horowitz. Surface Micromachined Z-Axis Vibratory Rate Gyroscope. Proc. Solid-State Sensors and Actuators, Hilton Head, SC, June 1996.

[4] S. Park and R. Horowitz. Adaptive Control for Z-Axis MEMS Gyroscopes. American Control Conference, Arlington, VA, June 2001.

[5] R.P. Leland. Adaptive Tuning for Vibrational Gyroscopes. Proceedings of IEEE Conference on Decision and Control, Orlando, FL, Dec. 2001.

[6] C. Acar, A. Shkel. Four Degrees-of-Freedom Micromachined Gyroscopes. Journal of Modeling and Sim. of Microsystems, Vol. 2, pp. 71-82, 2001.

[7] C. Acar, A. Shkel. Non-Resonant Micromachined Gyroscopes with Structural Mode-Decoupling. IEEE Sensors Journal, Vol. 3, No. 4, pp. 497-506, 2003.

[8] C. Acar, A. Shkel. Distributed-Mass Micromachined Gyroscopes for Enhanced Mode-Decoupling. IEEE Sensors Conference, September 2003, Toronto, Canada.

[9] J.A. Geen. A Path to Low Cost Gyroscopy. Solid-State Sensor and Actuator Workshop, Hilton-Head, SJ, 1998, pp. 51-54.

[10] W. Geiger, et.al. Decoupled Microgyros and the Design Principle DAVED. IEEE Sensors Journal, 2001, pp. 170-173.

[11] S.E. Alper, and T. Akin. A Symmetric Surface Micromachined Gyroscope with Decoupled Oscillation Modes. Sensors and Actuators A, Vol. 97, 2002, pp. 347-358. 